\title{
Consumption of kiwifruit capsules increases Faecalibacterium prausnitzii abundance in functionally constipated individuals: a randomised controlled human trial
}

\author{
Paul Blatchford ${ }^{1}$, Halina Stoklosinski ${ }^{1}$, Sarah Eady $^{2}$, Alison Wallace ${ }^{2}$, Christine Butts ${ }^{1}$, Richard Gearry ${ }^{3}$, \\ Glenn Gibson ${ }^{4}$ and Juliet Ansell ${ }^{1}$ \\ ${ }^{1}$ The New Zealand Institute for Plant \& Food Research Limited, Private Bag 11600, Palmerston North 4442, New Zealand \\ ${ }^{2}$ The New Zealand Institute for Plant \& Food Research Limited, Private Bag 4704, Lincoln 8140, New Zealand \\ ${ }^{3}$ Department of Medicine, University of Otago, Christchurch, New Zealand \\ ${ }^{4}$ Department of Food and Nutritional Sciences, University of Reading, Reading RG6 6AP, UK
}

(Received 23 June 2017 - Final revision received 19 July 2017 - Accepted 1 August 2017)

Journal of Nutritional Science (2017), vol. 6, e52, page 1 of 10

doi:10.1017/jns.2017.52

Abstract

This study investigated the impact of ACTAZIN ${ }^{\mathrm{TM}}$ green $(2400$ and $600 \mathrm{mg})$ and Livaux ${ }^{\mathrm{TM}}(2400 \mathrm{mg})$ gold kiwifruit supplements on faecal microbial composition and metabolites in healthy and functionally constipated (FC) participants. The participants were recruited into the healthy group ( $n$ 20; one of whom did not complete the study) and the FC group $(n 9)$, each of whom consumed all the treatments and a placebo (isomalt) for 4 weeks in a randomised cross-over design interspersed with 2-week washout periods. Modification of faecal microbiota composition and metabolism was determined by $16 \mathrm{~S}$ rRNA gene sequencing and GC, and colonic $\mathrm{pH}$ was calculated using SmartPill ${ }^{\mathbb{B}}$ wireless motility capsules. A total of thirty-two taxa were measured at greater than $1 \%$ abundance in at least one sample, ten of which differed significantly between the baseline healthy and FC groups. Specifically, Bacteroidales and Roseburia spp. were significantly more abundant $(P<0 \cdot 05)$ in the healthy group and taxa including Ruminococcaceae, Dorea spp. and Akekrmansia spp. were significantly more abundant $(P<0.05)$ in the FC group. In the FC group, Faecalibacterium prausnitzii abundance significantly increased $(P=0.024)$ from 3.4 to $7.0 \%$ following Livaux ${ }^{\mathrm{TM}}$ supplementation, with eight of the nine participants showing a net increase. Lower proportions of $F$. prausnitzii are often associated with gastrointestinal disorders. The discovery that Livaux ${ }^{\mathrm{TM}}$ supplementation increased F. prausnitzii abundance offers a potential strategy for improving gut microbiota composition, as F. prausnitziii is a butyrate producer and has also been shown to exert anti-inflammatory effects in many studies.

Key words: Kiwifruit: Gut microbiota: Human studies: Constipation: Faecalibacterium prausnitzii

The gastrointestinal tract harbours approximately $10^{14}$ microbial cells, consisting of over 1000 species or phylotypes, the majority of which reside in the colon ${ }^{(1,2)}$. The colonic microbiota has been associated with a wide range of health benefits including improved immune function and maturation, modified behaviour, regulation of satiety, inhibition of pathogens and augmentation of mineral absorption ${ }^{(3-5)}$. Imbalances in microbial community composition may be caused by diet, genetics, age, stress or xenobiotics and can induce a state of dysbiosis that may promote a more disease-susceptible microbiota. Consumption of certain types of dietary components can have a major influence on the communities of colonic microorganisms, with changes typically being observed within $24 \mathrm{~h}$ of consumption ${ }^{(6,7)}$.

Abbreviations: FC, functionally constipated; H, high dose; L, low dose.

* Corresponding author: Juliet Ansell, present address Zespri International Limited, 400 Maunganui Road, Mount Maunganui, Tauranga 3116 , New Zealand, fax +64 7 572 7646, email juliet.ansell@zespri.com 
Kiwifruit has been implicated in promoting several aspects of gut health including improving stool frequency, increasing commensal bacteria and improving aspects of immune function $^{(8-10)}$. These benefits are thought to derive from the inherent levels of digestion-resistant carbohydrates, polyphenols and vitamin $C^{(11)}$. The laxative effect of kiwifruit is undoubtedly the most well-studied health impact, whereas prebiotic effects are less well understood. In addition to host absorption of nutrients, enhancement of colonic microbial composition is thought to mediate many of the beneficial health effects of kiwifruit. Organic acids such as acetate, propionate and butyrate are produced as a result of microbial fermentation of kiwifruit digestion-resistant carbohydrates ${ }^{(9)}$. Organic acids may be absorbed by colonocytes into the bloodstream where they are further metabolised in the liver, muscle and other peripheral tissues, and are associated with the mediation of immune effects, cholesterol, satiety, and improved mineral absorption ${ }^{(12-14)}$.

As the importance of a robust and diverse colonic microbiota is increasingly apparent and the potential of kiwifruit to modulate the microbiota is being unearthed, a human intervention study was conducted to ascertain the effect of kiwifruit-derived supplements on colonic microbial composition and metabolism. Two kiwifruit-derived supplements, ACTAZIN ${ }^{\text {TM }}$ green-fleshed (Actinidia chinensis var. deliciosa 'Hayward') and Livaux ${ }^{\mathbf{T M}}$ gold-fleshed (Actinidia chinensis var. chinensis 'Zesy002') (Anagenix Ltd), were used as dietary interventions in the trial. These capsules are cold-processed dietary supplements, from which the skin and seeds are removed, formulated to maintain the integrity of innate kiwifruit compounds. The objective of this study was to assess the impact of ACTAZINTM and Livaux ${ }^{\mathrm{TM}}$ on the composition of colonic bacteria, metabolites and gut $\mathrm{pH}$, which was measured by $16 \mathrm{~S}$ rRNA gene sequencing, organic acid analysis and SmartPill ${ }^{\circledR}$ technology, respectively.

\section{Materials and methods}

\section{Study protocol}

The study design was a randomised double-blind placebocontrolled cross-over trial with participants consuming four different interventions for 4 weeks each, with a 2 -week washout between each intervention. A schematic view of the trial design is shown in Supplementary Fig. S1. The interventions were delivered in $4 \times 600 \mathrm{mg}$ capsules supplied by Anagenix Ltd prepared to look identical to preserve intervention blinding (Table 1). Detailed information on ingredient composition, trial recruitment and participant inclusion and exclusion criteria can be found in the Ansell et al. ${ }^{(15)}$ paper reporting laxation endpoints of this trial. Briefly, participants consumed four different intervention combinations: placebo (isomalt coloured green) (2400 mg/d), ACTAZIN ${ }^{\text {TM }}$ L $(600 \mathrm{mg} / \mathrm{d})$, ACTAZIN $^{\text {TM }} \mathrm{H}$ $(2400 \mathrm{mg} / \mathrm{d})$ and Livaux $^{\text {TM }}(2400 \mathrm{mg} / \mathrm{d})$ for $28 \mathrm{~d}$ each intervention, with a $14 \mathrm{~d}$ washout period between each treatment phase. ACTAZIN'M L (low dose, green kiwifruit) and ACTAZIN'TM H (high dose, green kiwifruit) were formulated from cold-processed Actinidia chinensis var. deliciosa 'Hayward' green kiwifruit and Livaux ${ }^{\mathrm{TM}}$ was formulated from coldprocessed Actinidia chinensis var. chinensis 'Zesy002' gold-fleshed kiwifruit. The placebo ingredient was isomalt (1-O- $\alpha$-Dglucopyranosyl-D-mannitol). Subjects were asked to exclude high-fibre dietary supplements such as Metamucil, Benefibre and Phloe as well as maintaining their habitual dietary intakes and physical activity habits and to refrain from eating fresh kiwifruit for the study period. At the beginning and end of each 4-week intervention period, participants were asked to provide a faecal sample. The washout period of 2 weeks was chosen to allow sufficient time to return bowel habits to baseline for the parameters measured (microbial ecology, microbial metabolites and SmartPill ${ }^{\circledR} \mathrm{pH}$ measurements). This study was conducted according to the guidelines laid down in the Declaration of Helsinki and all procedures involving human subjects/patients were approved by the New Zealand Human Disability and Ethics Committee (application number 12/ STH/72/AM01). Written informed consent was obtained from all subjects/patients. The trial was registered with the Australia New Zealand Clinical Trials Registry (registration number ACTRN: 12612001270808) (http://www.anzctr.org.au/).

\section{DNA extraction and 165 rRNA gene sequencing}

All faecal samples were collected and stored at $-20^{\circ} \mathrm{C}$ until analysis. Each sample $(250 \mathrm{mg})$ was weighed into a sterile microtube and DNA extracted using the MO-BIO PowerSoil $^{\circledR}$ DNA Isolation Kit (catalogue no. 12888; MO-BIO Laboratories). Illumina MiSeq $16 \mathrm{~S}$ rRNA gene sequencing was performed as described in a previous study ${ }^{(16)}$.

\section{Bioinformatics}

Quantitative Insights Into Microbial Ecology (QIIME) software version 1.8.0 was used to analyse the Illumina MiSeq sequencing data ${ }^{(17)}$. To assemble paired-end reads into a single continuous sequence, PANDASeq was used with parameters of at least $40 \mathrm{bp}$ overlap, a minimum of $350 \mathrm{bp}$ length and maximum of $500 \mathrm{bp}$ length ${ }^{(18)}$. Putative chimeras were filtered from the sequences and the reads clustered into operational taxonomic units based on a $97 \%$ identity threshold value using USEARCH and UCLUST ${ }^{(19)}$. A subsample of the

Table 1. Description of intervention composition

\begin{tabular}{llcl}
\hline Intervention & \multicolumn{1}{c}{ Treatment } & Dose $(\mathrm{mg})$ & Delivery - capsules/d \\
\hline ACTAZIN $^{\mathrm{TM}} \mathrm{L}$ & Green kiwifruit powder & 600 & $1 \times \mathrm{ACTAZIN}^{\mathrm{TM}}+3 \times$ placebo \\
ACTAZIN $^{\mathrm{TM}} \mathrm{H}$ & Green kiwifruit powder & 2400 & $4 \times$ ACTAZIN $^{\mathrm{TM}}$ \\
Livaux $^{\mathrm{TM}}$ & Gold kiwifruit powder & 2400 & $4 \times$ Livaux $^{\mathrm{TM}}$ \\
Placebo & Isomalt coloured green (E102, E142) & 2400 & $4 \times$ placebo \\
\hline
\end{tabular}

L, low dose; $\mathrm{H}$, high dose. 
total reads was taken to allow faster processing of the samples and to normalise at approximately 15000 reads per sample, which is sufficient for phylogenetic and taxonomic assignment ${ }^{(20,21)}$. Alignment of the sequences was carried out using PyNAST ${ }^{(2)}$ with reference to the Greengenes core reference database (version 13_8) ${ }^{(23)}$. Taxonomic assignment was made using the Ribosomal Database Project (RDP) naive Bayesian classifier ${ }^{(24)}$. Healthy and functionally constipated (FC) groups were analysed separately and the effect of each of the four treatments on microbial community composition determined by comparing the average abundance of each bacterial genus following treatment (greater than $1 \%$ abundance in at least one of the eight samples) with the average value before intervention.

\section{Organic acid quantification by GC}

A 500-1000 mg portion of each faecal sample was weighed into a clean tube and diluted 1:10 in PBS. An internal standard (ethyl butyrate) was included to give a final concentration of $5 \mathrm{~mm}$. Organic acids were quantified by GC using a modified method ${ }^{(25)}$. Analysis was performed on a Shimadzu GC system (GC-17A) equipped with a flame ionisation detector and fitted with an HP-1 column (Agilent Technologies). The instrument was controlled and chromatograms acquired using GC Solution Chromatography Data System software (Shimadzu, version 2.3). Organic acid concentrations were expressed as $\mu \mathrm{mol} / \mathrm{g}$ faeces.

\section{SmartPill ${ }^{\oplus}$}

SmartPill ${ }^{\circledR}$ is a single-use, ingestible capsule and used in clinical medicine as an alternative approach for determining gastrointestinal transit time ${ }^{(26)}$ instead of using radio-opaque markers and scintigraphy. SmartPill ${ }^{\circledR}$ is able to measure in situ gastrointestinal $\mathrm{pH}$, pressure and temperature and uses telemetry to relay information wirelessly to a small receiving device worn during the study. Six participants from the FC group underwent SmartPill ${ }^{\circledR}$ testing whilst on either the placebo or ACTAZINTM $H$ treatments. SmartPill ${ }^{\circledR}$ data were compared between treatments to ascertain whether ingestion of ACTAZINTM $\mathrm{H}$ capsules had an impact on $\mathrm{pH}$. Migration of the SmartPill ${ }^{\mathbb{R}}$ capsule past the ileocaecal junction into the proximal colon was characterised by a distinct decline in $\mathrm{pH}$. The entire colonic $\mathrm{pH}$ from the ileocaecal junction to passing the capsule was averaged and compared between treatments for all participants. Faecal samples originate from the distal bowel, therefore distal rectosigmoid colon $\mathrm{pH}$ was determined by taking the last $35.4 \%$ of total colonic $\mathrm{pH}$ measurements as calculated in a study interpreting segmental colonic transit times ${ }^{(27)}$. Bacterial groups were correlated with distal colonic $\mathrm{pH}$ data from SmartPill ${ }^{\circledR}$ measurements. A graphical example of SmartPill ${ }^{\circledR}$ data output for a participant is shown in Supplementary Fig. S2.

\section{Statistical analysis}

Statistical calculations were conducted in RStudio using the statistics package ${ }^{(28)}$. Bacterial groups were correlated with rectosigmoid $\mathrm{pH}$ data from SmartPill ${ }^{\circledR}$ measurements using the Spearman's rank correlation calculation. The Wilcoxon signed rank test was performed to assess significant differences between taxa and significant differences between organic acid concentrations before and after each treatment. A $P$ value of less than 0.05 was deemed significant after correcting for multiple comparisons using the false discovery rate (FDR) method in the p.adjust function in RStudio ${ }^{(29)}$.

\section{Results}

\section{5 rRNA gene sequencing}

High-throughput sequencing of variable regions of the $16 \mathrm{~S}$ rRNA gene, amplified from faecal sample-derived bacterial DNA, resulted in 26.3 million reads. After quality filtering, chimera removal and subsampling, a total of 3.72 million reads were obtained at an average of 14879 (14139 minimum-14 999 maximum) sequences per sample. Over all samples, 218 species-level phylotypes were observed at a $97 \%$ sequence identity threshold.

Analysis of the participants' faecal microbiota composition before intervention showed marked differences between the healthy and FC cohorts. A total of thirty-two genera were measured at greater than $1 \%$ abundance in at least one sample, ten of which differed significantly between the baseline healthy and FC groups (Fig. 1). Furthermore, the ratio of Firmicutes to Bacteroidetes differed between the groups at 2.25 and 3.19 for the healthy and FC groups, respectively. These numbers agree with other studies that show an increase in Firmicutes in irritable bowel syndrome patients relative to healthy controls ${ }^{(30)}$.

In the healthy group, Clostridiales increased significantly after Livaux ${ }^{\mathrm{TM}}$ supplementation from 5.0 to $7.6 \%(P=$ 0.042) (Table 2). In the FC group, Dorea spp. increased from 0.9 to $1.4 \%(P=0.008)$ after the ACTAZIN ${ }^{\text {TM }} \mathrm{H}$ treatment (Table 3). In the FC group, Faecalibacterium prausnitzii significantly increased after Livaux ${ }^{\mathrm{TM}}$ treatment from $3 \cdot 4$ to $7 \cdot 0$ $\% \quad(P=0.024)$, a two-fold increase as can be seen in Table 3. Individual participant responses can be seen in Supplementary Fig. S3, where eight out of the nine participants exhibited a net increase in F. prausnitzii.

\section{Organic acid production}

Succinate significantly decreased in concentration from $2 \cdot 3$ to $1.7 \mu \mathrm{mol} / \mathrm{g}(P=0.040)$ following placebo treatment in the FC group (Fig. 3). There were no other significant alterations to organic acid concentrations with any of the treatments. Quantitative differences before and after each treatment were generally modest, except for acetate which increased or decreased by up to $13 \mu \mathrm{mol} / \mathrm{g}$ after some treatments (Figs 2 and 3).

\section{SmartPill ${ }^{\oplus}$}

SmartPill ${ }^{\circledR}$ data were used to determine the $\mathrm{pH}$ of the colonic environment in a small group of participants taking the ACTAZINTM $H$ capsules and the placebo treatment. 

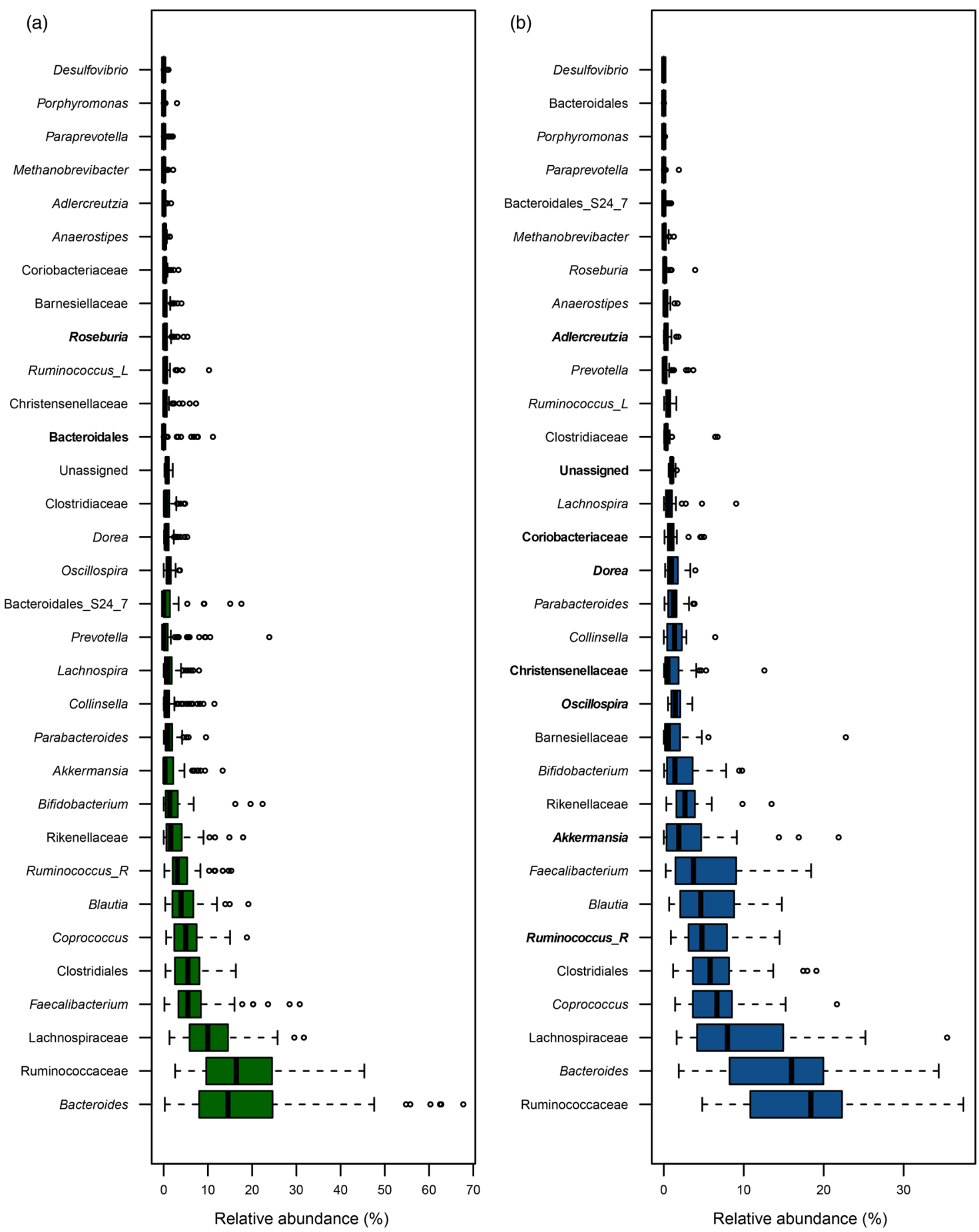

Fig. 1. Boxplots displaying bacterial taxa in healthy (a) and functionally constipated (b) baseline samples of faecal microbiota that are present at greater than $1 \%$ abundance in at least one sample. The genera in bold font are significantly more abundant compared with the other group, as calculated by the Wilcoxon signed rank test after false discovery rate correction for multiple comparisons $(P \leq 0.05)$. Ruminococcus_R, from Ruminococcaceae family; Ruminococcus_L, from Lachnospiraceae family.

Comparable $\mathrm{pH}$ values were observed between treatments, with an average $\mathrm{pH}$ of 6.38 in the ACTAZIN H treatment and an average $\mathrm{pH}$ of 6.30 in the placebo treatment (Supplementary Table S4). A Spearman rank correlation between bacterial groups and rectosigmoid $\mathrm{pH}$ provided two significant values: Bifidobacterium spp. negatively correlated with $\mathrm{pH}(-0.41 ; P=0.044)$ and Coprococcus spp. positively correlated with $\mathrm{pH}(0.43 ; P=0.039)$.

\section{Discussion}

Other results arising from this trial, assessing laxation endpoints, were published separately and showed that the kiwifruit supplements were able to significantly increase bowel movements in healthy individuals ${ }^{(15)}$. There is a strong relationship between stool consistency and microbiota composition and diversity $^{(31,32)}$, which is unsurprising as microbial metabolic end 
Table 2. Relative abundance of prevalent bacterial groups in response to treatments in the healthy group $\dagger$

\begin{tabular}{|c|c|c|c|c|c|c|c|c|c|c|c|c|c|c|c|c|}
\hline & \multicolumn{4}{|c|}{ Placebo } & \multicolumn{4}{|c|}{ Livaux $^{\mathrm{TM}}$} & \multicolumn{4}{|c|}{ ACTAZINTM L } & \multicolumn{4}{|c|}{ ACTAZIN ${ }^{\top M} \mathrm{H}$} \\
\hline & \multicolumn{2}{|c|}{ Pre } & \multicolumn{2}{|c|}{ Post } & \multicolumn{2}{|c|}{ Pre } & \multicolumn{2}{|c|}{ Post } & \multicolumn{2}{|c|}{ Pre } & \multicolumn{2}{|c|}{ Post } & \multicolumn{2}{|c|}{ Pre } & \multicolumn{2}{|c|}{ Post } \\
\hline & Mean & SEM & Mean & SEM & Mean & SEM & Mean & SEM & Mean & SEM & Mean & SEM & Mean & SEM & Mean & SEM \\
\hline Bacteroides & 18.8 & 4.0 & 18.0 & $3 \cdot 2$ & $23 \cdot 1$ & 4.6 & 22.0 & 4.2 & $16 \cdot 7$ & 3.1 & 18.4 & 3.9 & $19 \cdot 4$ & $3 \cdot 2$ & $18 \cdot 1$ & 3.8 \\
\hline Ruminococcaceaeł & $16 \cdot 3$ & $2 \cdot 4$ & $19 \cdot 6$ & 3.0 & 17.4 & 2.3 & 17.8 & 2.5 & 19.9 & 2.5 & $22 \cdot 2$ & 3.8 & 18.4 & 2.6 & 19.9 & 3.3 \\
\hline Lachnospiraceaeł & $10 \cdot 8$ & 1.3 & 11.0 & 1.5 & 11.9 & 1.9 & 9.5 & 1.3 & 11.6 & 1.6 & $9 \cdot 3$ & 1.7 & 11.6 & 1.6 & 11.0 & 1.4 \\
\hline Faecalibacterium & 7.0 & 1.5 & 5.7 & 1.0 & $7 \cdot 2$ & 1.6 & 5.3 & 0.9 & $6 \cdot 3$ & 1.6 & 4.6 & 0.8 & 6.7 & $1 \cdot 1$ & 6.5 & 1.6 \\
\hline Clostridialesł & 5.9 & 0.9 & 5.7 & 1.0 & 5.0 & 0.9 & $7 \cdot 6^{\star}$ & $1 \cdot 1$ & 5.9 & 0.7 & 5.0 & $1 \cdot 1$ & 6.6 & $1 \cdot 2$ & 5.6 & 0.9 \\
\hline Coprococcus & 6.6 & 1.0 & 5.5 & 0.8 & 5.9 & $1 \cdot 1$ & $5 \cdot 1$ & 0.7 & 5.4 & 0.8 & $5 \cdot 2$ & 0.8 & $5 \cdot 1$ & 0.8 & 4.9 & 0.8 \\
\hline Blautia & 5.8 & 0.9 & 4.7 & 1.0 & 4.3 & 0.6 & 4.8 & 1.0 & 4.7 & 0.7 & 6.3 & 1.7 & 4.8 & $1 \cdot 1$ & 6.1 & 1.8 \\
\hline Ruminococcus_R $R$ & 4.4 & 1.0 & 5.9 & 1.6 & 3.8 & 0.6 & 3.7 & $1 \cdot 0$ & $5 \cdot 3$ & 1.0 & 5.9 & 1.3 & 3.5 & 0.8 & 4.4 & $1 \cdot 2$ \\
\hline Rikenellaceaeł & 2.8 & 0.9 & 2.7 & 0.7 & 2.7 & 0.7 & 2.9 & 1.0 & 2.8 & 1.0 & 2.5 & 0.9 & 3.0 & 0.6 & 3.5 & 0.8 \\
\hline Bifidobacterium & 3.0 & 1.0 & $2 \cdot 3$ & 0.6 & 2.5 & 1.2 & $2 \cdot 1$ & 0.6 & $2 \cdot 0$ & 0.4 & 3.5 & 1.1 & $2 \cdot 7$ & 0.9 & $2 \cdot 6$ & 0.8 \\
\hline Bacteroidales $\ddagger$ & $2 \cdot 2$ & 1.0 & $2 \cdot 2$ & 0.8 & 2.0 & 0.9 & 1.9 & 0.8 & 1.3 & 0.4 & 1.2 & 0.3 & 2.6 & 1.1 & 1.7 & 0.6 \\
\hline Lachnospira & 1.6 & 0.5 & 1.1 & 0.2 & 1.3 & 0.3 & 1.5 & 0.5 & 1.0 & 0.2 & 1.1 & 0.3 & 1.8 & 0.5 & 1.3 & 0.4 \\
\hline Parabacteroides & 1.2 & 0.3 & 1.6 & 0.5 & 1.8 & 0.6 & 1.1 & 0.2 & 1.3 & 0.3 & 1.0 & 0.2 & 1.7 & 0.3 & 1.5 & 0.3 \\
\hline Collinsella & 1.8 & 0.7 & 1.2 & 0.4 & 0.9 & 0.4 & 1.3 & 0.4 & 1.6 & 0.6 & 1.2 & 0.3 & 1.5 & 0.6 & $1 \cdot 1$ & 0.3 \\
\hline Akkermansia & 1.3 & 0.5 & 1.4 & 0.6 & 1.8 & 0.7 & 1.6 & 0.7 & 1.6 & 0.6 & 1.8 & 0.5 & 1.4 & 0.4 & 1.0 & 0.3 \\
\hline Oscillospira & 1.1 & 0.2 & 1.1 & 0.2 & 1.1 & 0.2 & 1.6 & 0.3 & 1.4 & 0.2 & 1.5 & 0.5 & 1.2 & 0.1 & 1.5 & 0.2 \\
\hline Prevotella & 1.5 & 0.7 & 2.6 & 1.8 & 0.8 & 0.4 & $2 \cdot 1$ & 1.5 & 2.5 & 1.33 & 0.7 & 0.3 & 0.9 & 0.5 & 1.6 & 0.9 \\
\hline Dorea & 1.2 & 0.4 & 1.2 & 0.3 & 0.9 & 0.2 & 1.5 & 0.4 & 1.1 & 0.2 & 0.9 & 0.2 & 0.8 & 0.2 & 1.0 & 0.2 \\
\hline Clostridiaceaeł & 1.5 & 0.4 & 0.9 & 0.3 & 0.9 & 0.2 & 0.9 & 0.2 & 0.8 & 0.2 & 1.2 & 0.4 & 0.8 & 0.2 & 0.9 & 0.3 \\
\hline Unassigned & 0.8 & 0.1 & 1.0 & 0.2 & 0.8 & 0.1 & 1.0 & 0.2 & 0.9 & 0.1 & 0.9 & 0.1 & 0.8 & 0.1 & 0.8 & 0.1 \\
\hline
\end{tabular}

, low dose; $\mathrm{H}$, high dose; Ruminococcus_R, from Ruminococcaceae family.

* Significantly different compared with pre-treatment based on the Wilcoxon signed rank test after false discovery rate correction for multiple comparisons $(P \leq 0.05)$.

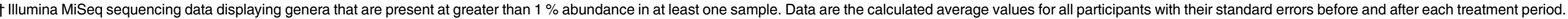
₹ Some bacteria could only be classified as far as the order or family level. 
Table 3. Relative abundance of prevalent bacterial groups in response to treatments in the functionally constipated group $\dagger$

\begin{tabular}{|c|c|c|c|c|c|c|c|c|c|c|c|c|c|c|c|c|}
\hline & \multicolumn{4}{|c|}{ Placebo } & \multicolumn{4}{|c|}{ Livaux $^{\mathrm{TM}}$} & \multicolumn{4}{|c|}{ ACTAZIN ${ }^{\top M} L$} & \multicolumn{4}{|c|}{ ACTAZINTM $\mathrm{H}$} \\
\hline & \multicolumn{2}{|c|}{ Pre } & \multicolumn{2}{|c|}{ Post } & \multicolumn{2}{|c|}{ Pre } & \multicolumn{2}{|c|}{ Post } & \multicolumn{2}{|c|}{ Pre } & \multicolumn{2}{|c|}{ Post } & \multicolumn{2}{|c|}{ Pre } & \multicolumn{2}{|c|}{ Post } \\
\hline & Mean & SEM & Mean & SEM & Mean & SEM & Mean & SEM & Mean & SEM & Mean & SEM & Mean & SEM & Mean & SEM \\
\hline Ruminococcaceaeł & $17 \cdot 2$ & $2 \cdot 3$ & 19.9 & 2.9 & $17 \cdot 1$ & 3.8 & $16 \cdot 3$ & $2 \cdot 1$ & 17.9 & 2.6 & $15 \cdot 0$ & 2.3 & 16.5 & 3.1 & $18 \cdot 8$ & $2 \cdot 1$ \\
\hline Bacteroides & $12 \cdot 6$ & 3.1 & 14.4 & 2.2 & 14.2 & 2.6 & 15.5 & 2.6 & $13 \cdot 9$ & $2 \cdot 2$ & $12 \cdot 2$ & 2.5 & 14.0 & 2.9 & $16 \cdot 3$ & $3 \cdot 1$ \\
\hline Lachnospiraceaeł & 11.4 & 1.6 & 8.0 & $2 \cdot 3$ & $10 \cdot 0$ & 2.5 & 13.9 & $3 \cdot 3$ & $10 \cdot 8$ & $3 \cdot 1$ & $11 \cdot 0$ & $2 \cdot 3$ & 11.7 & 2.5 & 8.7 & $2 \cdot 2$ \\
\hline Faecalibacterium & 7.9 & $2 \cdot 8$ & 5.8 & $2 \cdot 1$ & 3.4 & 1.0 & $7 \cdot 0^{*}$ & 1.1 & 4.9 & 1.7 & 5.0 & 1.4 & 7.7 & $2 \cdot 1$ & 3.1 & 1.0 \\
\hline Clostridiales $\ddagger$ & 4.8 & 0.6 & 6.8 & 1.7 & $6 \cdot 4$ & 1.3 & 5.5 & 1.1 & $6 \cdot 4$ & 1.2 & $6 \cdot 7$ & 1.7 & 6.0 & 1.4 & 7.5 & 1.8 \\
\hline Coprococcus & 6.5 & 1.4 & $6 \cdot 1$ & 1.1 & $6 \cdot 7$ & 1.7 & $6 \cdot 6$ & 1.4 & 6.9 & 1.4 & $8 \cdot 6$ & 1.2 & $6 \cdot 0$ & 1.2 & $7 \cdot 1$ & 1.9 \\
\hline Blautia & 6.6 & 1.8 & 3.5 & 0.9 & $7 \cdot 1$ & $2 \cdot 0$ & 6.0 & 1.5 & 6.9 & 1.5 & 7.5 & 1.8 & 5.9 & $2 \cdot 1$ & 5.4 & 1.3 \\
\hline Ruminococcus_R $R$ & 9.4 & 2.6 & $5 \cdot 1$ & 1.0 & 5.7 & 1.4 & 4.9 & 0.7 & 5.5 & $2 \cdot 0$ & $6 \cdot 7$ & 1.5 & $5 \cdot 1$ & $1 \cdot 1$ & 6.6 & 1.3 \\
\hline Bifidobacterium & 2.6 & 1.3 & 2.6 & 1.0 & 3.5 & 1.5 & 2.6 & 1.0 & $2 \cdot 7$ & 1.5 & $2 \cdot 7$ & 0.9 & 4.0 & 1.6 & 1.8 & 0.8 \\
\hline Akkermansia & 2.7 & 1.7 & 4.8 & 2.0 & $5 \cdot 0$ & 1.8 & 2.6 & 1.0 & 3.3 & $1 \cdot 1$ & 3.3 & 1.5 & 3.6 & 1.0 & 5.6 & $2 \cdot 3$ \\
\hline Rikenellaceaeł & $2 \cdot 3$ & 0.6 & $3 \cdot 1$ & 0.6 & 3.9 & $1 \cdot 2$ & $2 \cdot 4$ & 0.5 & 4.0 & $1 \cdot 1$ & 3.9 & 1.3 & $2 \cdot 6$ & 0.7 & 3.2 & 0.9 \\
\hline Christensenellaceae & 1.0 & 0.6 & 1.5 & 0.6 & $2 \cdot 2$ & 1.4 & $1 \cdot 1$ & 0.6 & 1.8 & 0.8 & 1.4 & 0.6 & $2 \cdot 3$ & 1.6 & $2 \cdot 2$ & 1.3 \\
\hline Prevotella & 1.8 & 1.1 & 0.6 & 0.3 & 0.2 & 0.1 & 0.5 & 0.3 & 0.5 & 0.3 & 0.9 & 0.5 & $2 \cdot 0$ & $1 \cdot 1$ & 0.2 & 0.1 \\
\hline Collinsella & 1.6 & 0.6 & $1 \cdot 2$ & 0.3 & 1.6 & 0.4 & 1.3 & 0.3 & 1.2 & 0.4 & 1.8 & 0.7 & 1.6 & 0.6 & 1.4 & 0.3 \\
\hline Parabacteroides & 1.3 & 0.4 & 1.3 & 0.4 & 1.2 & 0.3 & 1.3 & 0.4 & 1.7 & 0.4 & 1.2 & 0.4 & 1.3 & 0.4 & 1.5 & 0.3 \\
\hline Oscillospira & 1.3 & 0.1 & 1.5 & 0.3 & 1.6 & 0.2 & 1.8 & 0.3 & 1.6 & 0.2 & 1.5 & 0.3 & 1.3 & 0.2 & 1.8 & 0.3 \\
\hline Lachnospira & 1.0 & 0.5 & 1.0 & 0.4 & 0.6 & 0.2 & 1.6 & 0.9 & 1.3 & 0.5 & 1.2 & 0.5 & 1.3 & 0.6 & 0.5 & 0.1 \\
\hline Dorea & 1.5 & 0.4 & 1.4 & 0.3 & 1.3 & 0.3 & 1.2 & 0.3 & $1 \cdot 2$ & 0.3 & 1.2 & 0.4 & 0.9 & 0.2 & $1.4^{*}$ & 0.3 \\
\hline Coriobacteriaceaeł & 0.9 & 0.3 & 1.2 & 0.5 & 1.5 & 0.4 & 1.2 & 0.5 & 1.1 & 0.3 & $1 \cdot 1$ & 0.3 & 0.8 & 0.2 & $1 \cdot 2$ & 0.4 \\
\hline Barnesiellaceaeł & 0.9 & 0.3 & 4.0 & 2.4 & 1.0 & 0.4 & 1.0 & 0.3 & $1 \cdot 1$ & 0.3 & 1.4 & 0.5 & 0.8 & 0.3 & 0.8 & 0.3 \\
\hline Clostridiaceae & 0.4 & 0.1 & 1.5 & 0.7 & 0.9 & 0.5 & 1.0 & 0.7 & 0.3 & 0.1 & 0.4 & 0.1 & 0.4 & 0.1 & 0.3 & 0.1 \\
\hline Unassigned & 1.0 & 0.1 & $1 \cdot 1$ & 0.1 & 1.1 & 0.2 & 1.0 & 0.1 & 1.0 & 0.1 & 1.0 & 0.1 & 1.3 & 0.4 & 1.0 & 0.1 \\
\hline
\end{tabular}

$\mathrm{L}$, low dose; $\mathrm{H}$, high dose; Ruminococcus_R, from Ruminococcaceae family.

作

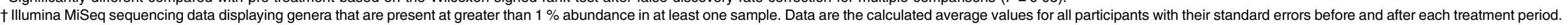

$\ddagger$ Some bacteria could only be classified as far as the order or family level. 
60
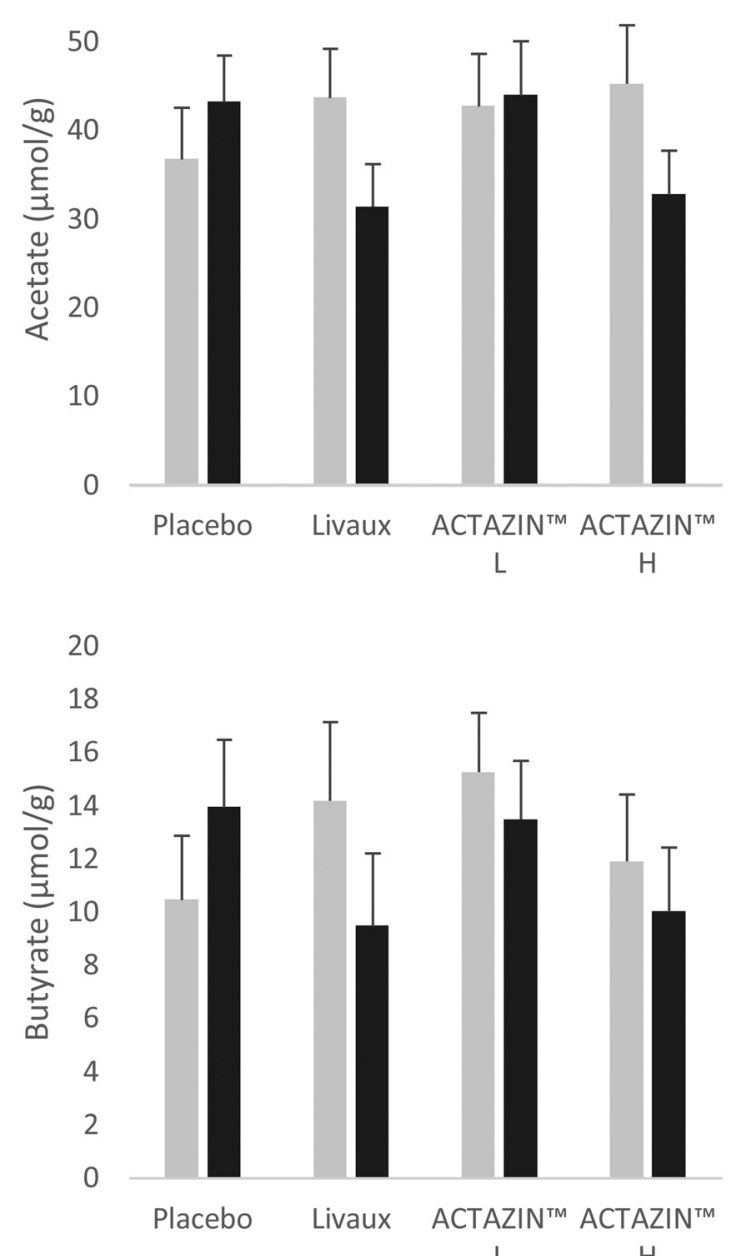

14

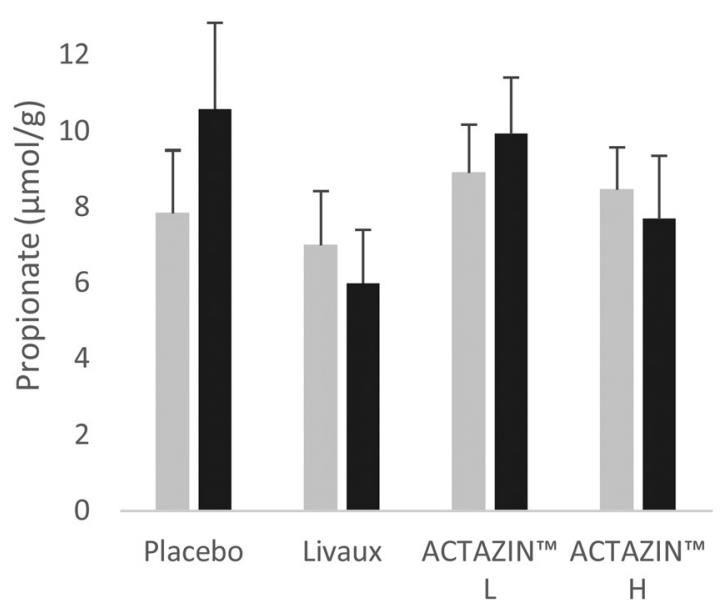

$5 \cdot 0$

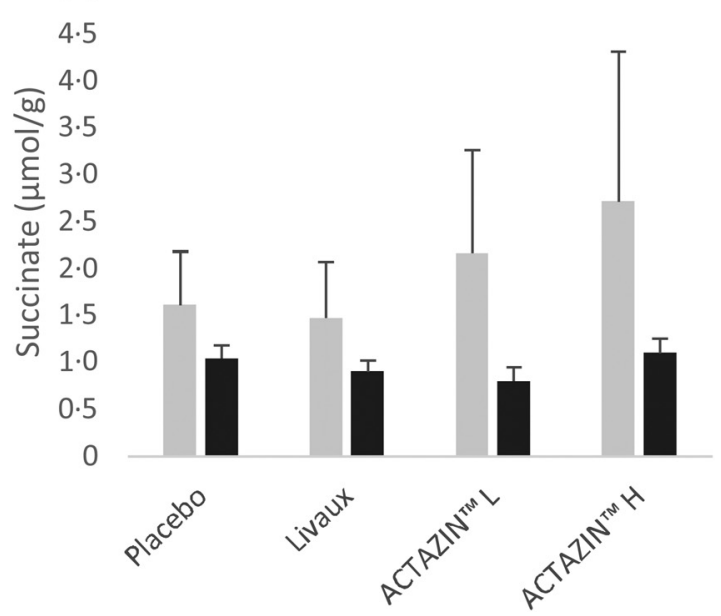

Fig. 2. Organic acid concentrations in faecal samples in response to the four treatments in the healthy group as measured by GC, expressed in $\mu$ mol/g faeces. :.: Pre-treatment; $\mathbf{\square}$, after treatment; L, low dose; $\mathrm{H}$, high dose. Values are means, with standard errors represented by vertical bars.

products such as SCFA and gases can affect colonic transit time ${ }^{(33,34)}$. However, in this trial no correlations were observed in those participants that exhibited an increase in stool frequency and microbiota composition. Comparison between the baseline microbiota composition of the healthy and FC groups has provided valuable insight to the bacterial aetiology of functional constipation. Interesting findings between the cohorts were a significantly higher Bacteroidales abundance in healthy individuals and significantly higher Akkermansia spp. abundance in FC participants. Both patterns agree with a study assessing microbiota composition and stool consistency ${ }^{(32)}$.

In the present study, we found that several bacterial groups were significantly altered in abundance following kiwifruit supplementation. Clostridiales increased by $2 \cdot 6 \%$ after Livaux ${ }^{\mathrm{TM}}$ supplementation in the healthy group, but as the order includes a wide range of families and genera, including Lachnospiraceae, Clostridiaceae, Ruminococcaceae, Veillonellaceae, etc. and some pathogens, it is difficult to draw meaningful conclusions about the consequence of this increase. Dorea spp. increased modestly after ACTAZIN H treatment in the FC group and these members of the diverse Lachnospiraceae family are major gas producers, mainly $\mathrm{CO}_{2}$ and $\mathrm{H}_{2}^{(35)}$.
A non-significant decrease in F. prausnitzii from 7.2 to $5.3 \%$ was observed in the healthy group which could be due to already high levels at baseline. Other studies have observed depleted F. prausnitzii in irritable bowel syndrome patients compared with healthy control subjects ${ }^{(30)}$. It has been demonstrated that the effect of baseline concentrations of a bacterial group may have a substantial bearing on the magnitude of effect observed in response to a dietary intervention ${ }^{(36,37)}$. F. prausnitzii abundance was significantly elevated (3.6\%) in the FC group after a 4-week period of supplementation with the gold kiwifruit-based Livaux $^{\mathrm{TM}}$. F. prausnitzii is one of the more populous species in the human gastrointestinal tract, being typically observed at over $5 \%$ of the total proportion of the colonic microbiota of healthy adults ${ }^{(38)}$, in agreement with abundance data presented in this study. Members of the Firmicutes phylum, F. prausnitzii are commensal inhabitants of the human large bowel, with demonstrated anti-inflammatory properties in vivo ${ }^{(39,40)}$. One study found that F. prausnitzii was able to utilise pectin and uronic acids as substrates for growth and both are known constituents of kiwifruit ${ }^{(41)}$. Green and gold kiwifruit have been shown to exhibit differences in digestibility, leading to altered soluble and insoluble fibre compositions reaching the large bowel ${ }^{(42)}$, 
60

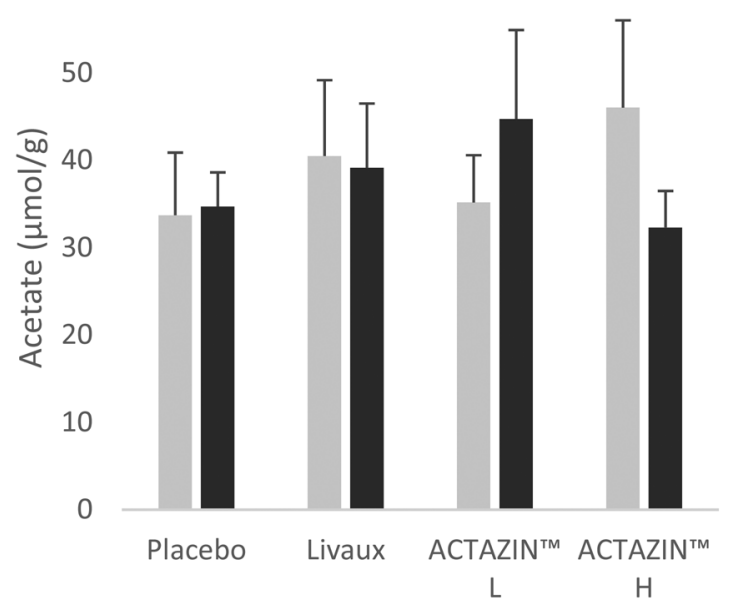

16

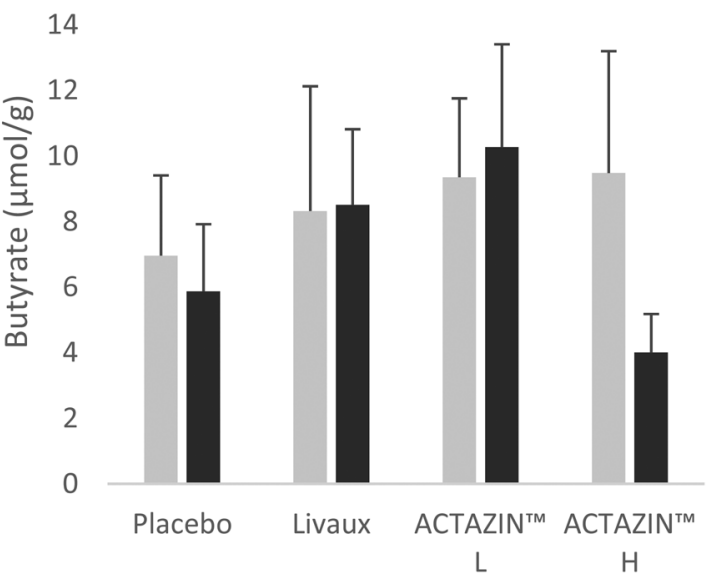

16

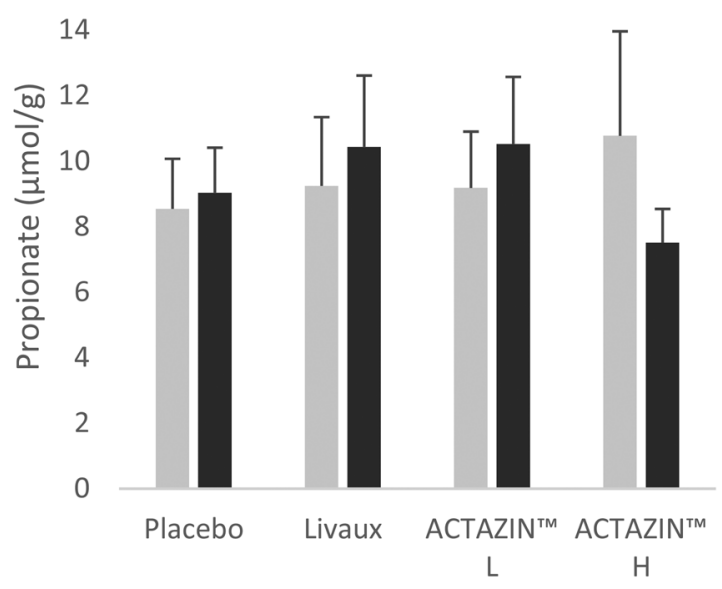

$3 \cdot 0$

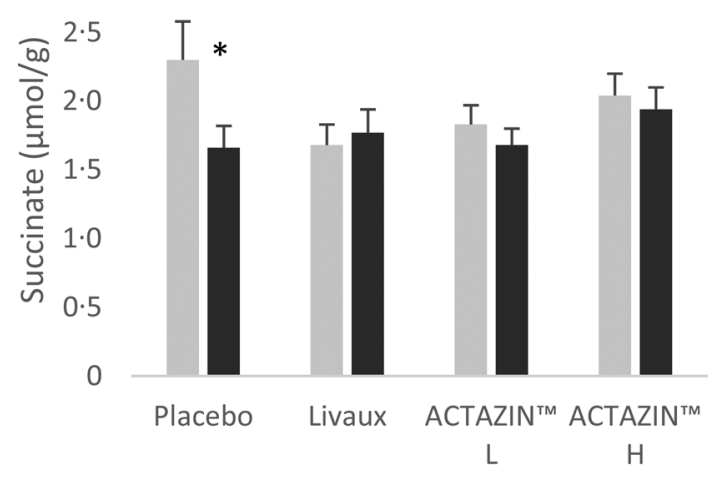

Fig. 3. Organic acid concentrations in faecal samples in response to the four treatments in the functionally constipated group as measured by GC, expressed in $\mu$ mol/ $\mathrm{g}$ faeces. : Pre-treatment; $\mathbf{\square}$, after treatment; L, low dose; $\mathrm{H}$, high dose. Values are means, with standard errors represented by vertical bars. ${ }^{\star}$ Significantly different compared with pre-treatment based on the Wilcoxon signed rank test after false discovery rate correction for multiple comparisons $(P \leq 0.05)$.

which may be why differences were observed in the effect of green and gold kiwifruit fibre in this study. Low amounts of $F$. prausnitzii have been associated with a range of intestinal disorders including irritable bowel syndrome, atopy, diabetes and inflammatory bowel diseases such as Crohn's disease and ulcerative colitis ${ }^{(30,39,40,43,44)}$. These consistent observations show that a depleted concentration of F. prausnitzii is an undesirable endpoint and therefore any treatment that can selectively stimulate its proliferation in situ is likely to be a promising intervention strategy.

The mechanisms by which F. prausnitzii facilitates its healthpromoting impact are most probably through butyrate production and subsequent anti-inflammatory effects. Butyrate is the preferred energy source for colonic epithelial cells and plays a role in alleviating inflammation as well as mitigating carcinogenesis, pathogenic colonisation and oxidative stress ${ }^{(12,45)}$. A mouse study by Sokol et al. ${ }^{(40)}$ found that F. prausnitzii or F. prausnitzii culture supernatant reduced the severity of chemical-induced colitis, promoted the synthesis of antiinflammatory cytokines and mitigated pro-inflammatory cytokine production, suggesting that anti-inflammatory effects are mediated by secreted metabolites. Therefore, increasing the amount of F. prausnitzii in the colon may help mitigate the symptoms of gastrointestinal disorders, potentially through elevated butyrate production.

There were minor differences between organic acids after treatments which could be attributed to using faecal samples as a proxy for in situ determination of organic acid composition and concentration. Organic acid concentrations decrease distally in the large bowel due to a number of reasons including secondary fermentation, absorption into the bloodstream and/or utilisation of organic acids (particularly butyrate) by colonocytes. Therefore, measurement of organic acids in faecal samples would greatly underestimate the concentration of in situ organic acids ${ }^{(46)}$. Despite these limitations, butyrate was slightly higher in faecal samples from participants after Livaux $^{\mathrm{TM}}$ treatment in the FC group, which aligns with the increased F. prausnitzii abundance but it was not to a significant level.

It has been demonstrated that $\mathrm{pH}$ can alter microbial populations ${ }^{(47)}$ and therefore it stands to reason that an altered $\mathrm{pH}$ in the colon could modify the colonic microbial profile. In the present study, correlating $\mathrm{pH}$ with bacterial groups has revealed one significantly positive and one significantly 
negative correlation. The discovery that Bifidobacterium spp. abundance was significantly higher at lower $\mathrm{pH}$ is an expected result as it is an acid-producing bacterium, and hence tolerant of such conditions. Increased bifidobacteria abundance is positive because it is recognised as an established probiotic genus, and in general a lower $\mathrm{pH}$ is seen as desirable as it can exclude the growth of competing pathogens ${ }^{(48)}$.

In conclusion, Livaux ${ }^{\mathrm{TM}}$ appears to stimulate proliferation of the commensal F. prausnitzii in participants with low initial F. prausnitzii concentrations and this may be associated with higher in situ butyrate concentrations, although this is difficult to measure in a human study. These results bear clinical relevance as stimulating an increase in an abundant butyrogenic bacterium could have beneficial consequences for ulcerative colitis patients. Future work to corroborate these results could entail in vitro studies to measure butyrate production and F. prausnitzii stimulation in a closed system.

\section{Supplementary material}

The supplementary material for this article can be found at https://doi.org/10.1017/jns.2017.52

\section{Acknowledgements}

We appreciate statistical review of this paper by Duncan Hedderley. We acknowledge the technical assistance of Nayer Ngametua and Genelle Healey with 16S rRNA gene sequencing sample preparation. ACTAZIN ${ }^{\mathrm{TM}}$ and Livaux ${ }^{\mathrm{TM}}$ were provided by Anagenix Ltd.

This study was funded from the New Zealand Ministry of Business, Innovation and Employment (Foods for Health at Different Life Stages programme, contract C11X1312), Anagenix Ltd and Stratum Nutrition (Saint Charles, MO, USA).

The authors have declared no conflict of interest.

\section{References}

1. Qin JJ, Li RQ, Raes J, et al. (2010) A human gut microbial gene catalogue established by metagenomic sequencing. Nature 464, $59-65$.

2. Rajilic-Stojanovic M \& de Vos WM (2014) The first 1000 cultured species of the human gastrointestinal microbiota. FEMS Microbiol Rev 38, 996-1047.

3. Buffie CG \& Pamer EG (2013) Microbiota-mediated colonization resistance against intestinal pathogens. Nat Rev Immunol 13, 790-801.

4. Geurts L, Neyrinck AM, Delzenne NM, et al. (2014) Gut microbiota controls adipose tissue expansion, gut barrier and glucose metabolism: novel insights into molecular targets and interventions using prebiotics. Benef Microbes 5, 3-17.

5. Parnell JA \& Reimer RA (2012) Prebiotic fibres dose-dependently increase satiety hormones and alter Bacteroidetes and Firmicutes in lean and obese JCR:LA-cp rats. Br J Nutr 107, 601-613.

6. Walker AW, Ince J, Duncan SH, et al. (2011) Dominant and diet-responsive groups of bacteria within the human colonic microbiota. ISME J 5, 220-230.

7. Wu GD, Chen J, Hoffmann C, et al. (2011) Linking long-term dietary patterns with gut microbial enterotypes. Science 334, 105-108.

8. Bentley-Hewitt KL, Blatchford PA, Parkar SG, et al. (2012) Digested and fermented green kiwifruit increases human $\beta$-defensin 1 and 2 production in vitro. Plant Foods Hum Nutr 67, 208-214.
9. Parkar SG, Rosendale D, Paturi G, et al. (2012) In vitro utilization of gold and green kiwifruit oligosaccharides by human gut microbial populations. Plant Foods Hum Nutr 67, 200-207.

10. Rush EC, Patel M, Plank LD, et al. (2002) Kiwifruit promotes laxation in the elderly. Asia Pac J Clin Nutr 11, 164-168.

11. Chan AO, Leung G, Tong $T$, et al. (2007) Increasing dietary fiber intake in terms of kiwifruit improves constipation in Chinese patients. World J Gastroenterol 13, 4771-4775.

12. Hamer HM, Jonkers D, Venema K, et al. (2008) Review article: the role of butyrate on colonic function. Aliment Pharmacol Ther 27, 104-119.

13. Saulnier DMA, Spinler JK, Gibson GR, et al. (2009) Mechanisms of probiosis and prebiosis: considerations for enhanced functional foods. Curr Opin Biotechnol 20, 135-141.

14. Wong JMW, de Souza R, Kendall CWC, et al. (2006) Colonic health: fermentation and short chain fatty acids. I Clin Gastroenterol 40, 235-243.

15. Ansell J, Butts CA, Paturi G, et al. (2015) Kiwifruit-derived supplements increase stool frequency in healthy adults: a randomized, double-blind, placebo-controlled study. Nutr Res 35, 401-408.

16. Blatchford P, Bentley-Hewitt KL, Stoklosinski H, et al. (2015) In vitro characterisation of the fermentation profile and prebiotic capacity of gold-fleshed kiwifruit. Benef Microbes 6, 829-839.

17. Caporaso JG, Kuczynski J, Stombaugh J, et al. (2010) QIIME allows analysis of high-throughput community sequencing data. Nat Methods 7, 335-336.

18. Masella AP, Bartram AK, Truszkowski JM, et al. (2012) PANDAseq: paired-end assembler for illumina sequences. BMC Bioinformatics 13, 31.

19. Edgar RC (2010) Search and clustering orders of magnitude faster than BLAST. Bioinformatics 26, 2460-2461.

20. Caporaso JG, Lauber CL, Walters WA, et al. (2011) Global patterns of $16 \mathrm{~S}$ rRNA diversity at a depth of millions of sequences per sample. Proc Natl Acad Sci U S A 108, Suppl. 1, 4516-4522.

21. Schloss PD, Schubert AM, Zackular JP, et al. (2012) Stabilization of the murine gut microbiome following weaning. Gut Microbes 3, 383-393.

22. Caporaso JG, Bittinger K, Bushman FD, et al. (2010) PyNAST: a flexible tool for aligning sequences to a template alignment. Bioinformatics 26, 266-267.

23. DeSantis TZ, Hugenholtz P, Larsen N, et al. (2006) Greengenes, a chimera-checked $16 \mathrm{~S}$ rRNA gene database and workbench compatible with ARB. Appl Environ Microbiol 72, 5069-5072.

24. Wang Q, Garrity GM, Tiedje JM, et al. (2007) Naive Bayesian classifier for rapid assignment of rRNA sequences into the new bacterial taxonomy. Appl Environ Microbiol 73, 5261-5267.

25. Richardson AJ, Calder AG, Stewart CS, et al. (1989) Simultaneous determination of volatile and non-volatile acidic fermentation products of anaerobes by capillary gas-chromatography. Lett Appl Microbiol 9, 5-8.

26. Timm D, Willis H, Thomas W, et al. (2011) The use of a wireless motility device $\left(\right.$ SmartPill $\left.{ }^{\mathbb{R}}\right)$ for the measurement of gastrointestinal transit time after a dietary fibre intervention. Br J Nutr 105, 1337-1342.

27. Metcalf AM, Phillips SF, Zinsmeister AR, et al. (1987) Simplified assessment of segmental colonic transit. Gastroenterology 92, 40-47.

28. RStudio (2012) RStudio: integrated development environment for $\mathrm{R}$ (version 0.97.551) [computer software]. Boston, MA. http:// www.rstudio.org/

29. Benjamini Y \& Hochberg Y (1995) Controlling the false discovery rate - a practical and powerful approach to multiple testing. $J$ Royal Stat Soc Series B Methodol 57, 289-300.

30. Rajilic-Stojanovic M, Biagi E, Heilig HG, et al. (2011) Global and deep molecular analysis of microbiota signatures in fecal samples from patients with irritable bowel syndrome. Gastroenterology 141, 1792-1801.

31. Gilbert JA \& Alverdy J (2016) Stool consistency as a major confounding factor affecting microbiota composition: an ignored variable? Gut 65, 1-2.

32. Vandeputte D, Falony G, Vieira-Silva S, et al. (2016) Stool consistency is strongly associated with gut microbiota richness and composition, enterotypes and bacterial growth rates. Gut 65, 57-62. 
33. Lewis SJ \& Heaton KW (1997) Increasing butyrate concentration in the distal colon by accelerating intestinal transit. Gut 41, 245-251.

34. Soares ACF, Lederman HM, Fagundes-Neto U, et al. (2005) Breath methane associated with slow colonic transit time in children with chronic constipation. J Clin Gastroenterol 39, 512-515.

35. Taras D, Simmering R, Collins MD, et al. (2002) Reclassification of Eubacterium formicigenerans Holdeman and Moore 1974 as Dorea formicigenerans gen. nov., comb. nov., and description of Dorea longicatena sp. nov., isolated from human faeces. Int J Syst Evol Microbiol 52, 423-428.

36. Kolida S, Meyer D \& Gibson GR (2007) A double-blind placebocontrolled study to establish the bifidogenic dose of inulin in healthy humans. Eur J Clin Nutr 61, 1189-1195.

37. Tuohy KM, Kolida S, Lustenberger AM, et al. (2001) The prebiotic effects of biscuits containing partially hydrolysed guar gum and fructo-oligosaccharides - a human volunteer study. Br J Nutr 86, 341-348.

38. Miquel S, Martin R, Rossi O, et al. (2013) Faecalibacterium prausnitzii and human intestinal health. Curr Opin Microbiol 16, 255-261.

39. Furet JP, Kong LC, Tap J, et al. (2010) Differential adaptation of human gut microbiota to bariatric surgery-induced weight loss: links with metabolic and low-grade inflammation markers. Diabetes 59, 3049-3057.

40. Sokol H, Seksik P, Furet JP, et al. (2009) Low counts of Faecalibacterium prausnitzii in colitis microbiota. Inflamm Bowel Dis 15, 1183-1189.

41. Lopez-Siles M, Khan TM, Duncan SH, et al. (2012) Cultured representatives of two major phylogroups of human colonic
Faecalibacterium prausnitzii can utilize pectin, uronic acids, and host-derived substrates for growth. Appl Environ Microbiol 78, $420-428$.

42. Carnachan SM, Bootten TJ, Mishra S, et al. (2012) Effects of simulated digestion in vitro on cell wall polysaccharides from kiwifruit (Actinidia spp.). Food Chem 133, 132-139.

43. Candela M, Rampelli S, Turroni S, et al. (2012) Unbalance of intestinal microbiota in atopic children. BMC Microbiol 12, 95.

44. Willing BP, Dicksved J, Halfvarson J, et al. (2010) A pyrosequencing study in twins shows that gastrointestinal microbial profiles vary with inflammatory bowel disease phenotypes. Gastroenterology 139, 1844-1854.e1.

45. Macfarlane GT \& Macfarlane S (2011) Fermentation in the human large intestine: its physiologic consequences and the potential contribution of prebiotics. J Clin Gastroenterol 45, Suppl., S120-S127.

46. Millet S, Van Oeckel MJ, Aluwe M, et al. (2010) Prediction of in vivo short-chain fatty acid production in hindgut fermenting mammals: problems and pitfalls. Crit Rev Food Sci Nutr 50, 605-619.

47. Walker AW, Duncan SH, McWilliam Leitch EC, et al. (2005) pH and peptide supply can radically alter bacterial populations and short-chain fatty acid ratios within microbial communities from the human colon. Appl Environ Microbiol 71, 3692-3700.

48. Sanchez B, Champomier-Verges MC, Collado Mdel C, et al. (2007) Low-pH adaptation and the acid tolerance response of Bifidobacterium longum biotype longum. Appl Environ Microbiol 73, 6450-6459. 